\title{
Bioorganic chemistry-a scientific endeavour in continuous transition
}

\author{
Nelson J. Leonard \\ Division of Chemistry and Chemical Engineering, The California \\ Institute of Technology, Pasadena, CA 91125
}

\begin{abstract}
Bioorganic chemistry is an expanding, dynamic area of fundamental and practical interest. The new technologies needed are developed through efficient organic synthesis and spectroscopic analysis or are assimilated from advances in biological manipulations. The multifaceted origins of bioorganic chemistry are discussed, along with the use of the descriptor in the titles of journals, organizations, and meetings. In the Second International Symposium on Bioorganic Chemistry, which is cosponsored by the International Union of Pure and Applied Chemistry and the Chemical Society of Japan and is hosted by Kyushu University, the topics to be covered indicate the accelerated pace and broadening scope of the enterprise.
\end{abstract}

We use the words "bioorganic chemistry" to describe what it is we do, or what it is we would like to do. It is not a new combination of words, but we ourselves may have realized only recently that our research interests do not end with organic chemistry -- structural. synthetic, or kinetic -- but that they now involve biological material. Structure guides us as to the potential details of how active biological partners may interact. Synthesis provides us with compounds that nature may not have created in sufficient quantity for investigation and also with analogues of natural species. Physical organic chenistry and analytical wethodology provide quantitative measures and intimate details of reaction pathways. In short. we use familiar principles and techniques toward solution of problems relevant to biology. In addition. we may be guided in our chemical studies by inspiration resulting from some tangential biological observation.

Bioorganic chemistry represents a merger of organic chemistry and biochemistry. In certain investigations, such as those involving metalloenzymes and cofactors, the contiguous areas of bioorganic and bioinorganic chemistry also merge. Even the composite term, biophysical organic chemistry, has been used as a detailed descriptor in molecular recognition. Bioorganic chemistry had multiple origins. Nutritional research identified factors essential in the human diet, and their structures and syntheses led to the recognition of the modes of action of the so-called vitamins and related cofactors, or coenzymes. Secreted factors that exert a stimulatory effect on cellular activity, the hormones, could be better understood at the molecular level once their structure determinations and syntheses made them available in reasonable amounts. Concepts of the biogenesis of natural products played, and continues to play, a major role in the development of bioorganic chemistry. The establishment of the complete stereochemical pathways of biosynthesis adds exquisiteness to this endeavour.

A simple chemical applied according to a well recognized concept can be responsible for a great advance in biological chemistry. Thus, through the reaction of cyanogen bromide, Bernhard Witkop (I) translated neighbouring group participation into selective, limited, non-enzymatic cleavage at methionine in a peptide chain. Application of the reagent has aided not only the correct sequencing of peptide segments of many proteins but also the production, through genetic engineering, of human insulin by means of a methionyl-containing precursor. Organic chemistry approaching $100 \%$ conversion at each. step provides the basis of modern biotechnology: the automated synthesis of polypeptide and polynucleotide chains and the sequencing of DNA and RNA. As organic chemists, we use chemically-based biotechnology and continue to add other techniques that are not only applicable but in some cases requisite: fluorescence sorting and probing; recombinant DNA technology; cloning; plasmid construction; 
combinatory procedures; the polymerase chain reaction (PCR); all of the latest separation and spectroscopic methodology with computer analysis; and the generous use -as reagents -- of bacteria, fungi, enzymes, whole cells, and ground liver microsomes, inter alia. A graduate course in bioorganic chemistry offered seven years ago would now be considered mainly historical. Even in two years' time, perhaps a third of any original material would have to be replaced by more recent findings. The frontiers of bioorganic chemistry are expanding at such a rate that wise exercise of selectivity in chemical approaches to the solution of biological problems will certainly be advantageous to present and future investigators.

Applications of the principles of physical organic chemistry led to important historical advances. A set of four examples from the research of Frank Westheimer and his coworkers (2) will serve to illustrate the point, starting with catalysis of the mutarotation of glucose by amino acids, which represented his first foray into the realm of enzymes. Later work included the classic study of hydride transfer to the coenzyme NAD+ from various substrates, which led to the recognition of enzyme stereospecificity. This was followed by the conception and verification of pseudorotation to account for the hydrolytic chemistry of phosphate esters and then by the first photoaffinity labeling experiment -- with diazoacetylchymotrypsin.

I am taking the liberty of including some examples from my own Laboratory that are not representative of sophisticated recognition of biological problems, as in the Westheimer examples. Instead, my examples can best be described as extrapolations from a chance observation during a natural product investigation. I shall omit details and references to myself and others in the interest of conserving time, except to mention that the investigation was initiated in our Laboratory in 1960. Triacanthine, the structure of which was established as $3-\left(\Delta^{2}-\right.$ isopentenyl)adenine, was isolated from the new leaves of the honey locust, Gleditsia triacanthos. Although it was inactive as a cytokinin, when it was autoclaved either alone or in culture medium, plant hormone activity developed, assayed by cell growth and differentiation of tobacco callus tissue. The active product of autoclaving, which was $N^{6}-\left(\Delta^{2}\right.$-isopentenyl)adenine, iPA, was also isolated from a number of natural sources including certain tRNAs, where it is found next to the anticodon. The path of rear-

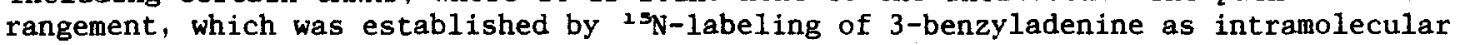
at least to the extent of $90 \%$, involves the opening and closure of both rings.

It was the synthesis of $3-\left(\Delta^{2}\right.$-isopenteny1)adenine and 3-benzyladenine that encouraged us to proceed to the synthesis of $3-(B-D-r i b o f u r a n o s y 1)$ adenine, 3-isoadenosine, and from this to the mono-, di-, and triphosphates. We recognized 3-iso-ATP as a spatial probe of enzyme-coenzyme interaction since superposition of the ring systems, with allowance for the preferred conformations at the ribosyl junction, shows remarkably similar loci for the respective sidechains and ring nitrogens. ATP and 3-iso-ATP showed comparable activity as coenzymes in a representative number of enzyme conversions (3). The lack of "visibility" of 3-iso-ATP as a probe was corrected by rendering ATP fluorescent by reaction with chloroacetaldehyde, with the formation of $1, N^{6}-$ ethenoadenosine 5'-triphosphate, $\varepsilon$-ATP, a fluorescent probe. Where this serves as a surrogate for ATP, the fluorescence properties -- yield, lifetime, and polarization - - are useful in the determination of the mode of coenzyme binding to enzymes and proteins. Moreover, the reaction of chloroacetaldehyde with unpaired adenine, guanine, and cytosine ribonucleotides and deoxyribonucleotides and selective synthetic incorporation of etheno( $\varepsilon)$-bridged nucleotides have broad diagnostic value in structure determination. These include tRNA conformational changes, tRNA-ribosome interactions, altered DNA conformations at specific sites, structural perturbations in supercoiled DNA, cruciform DNA structures, B- to Z-DNA transitions, misalignments in one DNA strand, and triple-helical DNA without and within cells. In addition, the detection and quantitation of $\varepsilon$-modified ribonucleotides has clarified the role of chloroacetaldehyde in carcinogenesis, especially where it is an intermediate in the microsomal activation of vinyl chloride and related carcinogens.

In order to provide a mimic of ATP that did not prevent binding at $\mathrm{N}$ and $\mathrm{NH}_{2}$ of the pyrimidine ring but retained the useful property of fluorescence, we synthesized linear-benzo-ATP, which has served as a fluorescent dinensional probe of coenzymeenzyme intraction. The stretching of the purine ring by a known magnitude, namely $2.4 \AA$, tested the dimensional restriction of enzyme-active sites. The inclusion of the property of fluorescence in these dimensional probes facilitated location of the binding sites as well as estimation of the flexibility for binding and activity of 
the dynamic reactive pair. With the finding that fragments of mitochondrial inner membranes phosphorylate 1 in-benzo-ADP and hydrolyze 1 in-benzo-ATP whereas intact mitochondria do not, it was possible to distinguish between transport selectivity and enzyme reactivity.

I have used our own chemistry merely to illustrate the point that it is also possible to adapt biological problems to the compounds that one has conceived and synthesized. Chemical probing techniques of this kind are achieving acceptance in molecular biology.

Let me return to a general duscussion of bioorganic chemistry that is more fitting for my present role in opening this International Symposium. There are laboratories with the title of bioorganic chemistry. For example, in Moscow the Shemyakin Institute for the Chemistry of Natural Products in 1974 became the Shemyakin Institute for Bioorganic Chemistry. The recent change in name to the Shemyakin-ovchinnikov Institute of Bioorganic Chemistry reminds us of its contributions to both protein and nucleic acid research. There are journal titles that include the word bioorganic, e.g., Bioorganic Chemistry (1971); Bioorganicheskaya Khimiya (1975); Bioorganic Marine Chemistry (1987); and Bioorganic \& Medicinal Chemistry Letters (1991), but bioorganic chemistry has become a mainstream science, with research articles appearing in all of the major journals.

The first IUPAC International Symposium on Bioorganic Chemistry was held in New York City in 1985 and was cosponsored by the New York Academy of Sciences. The idea for the initiation of these conferences came from Professor Jean Mathieu (France), who had promoted a successful EUCHEM Conference on Bioorganic Chemistry in 1983 and who was then the President of the Organic Chemistry Division of IUPAC. He convinced Ronald Breslow and Koji Nakanishi of Columbia University and the late Emil T. Kaiser of Rockefeller University to organize the first IUPAC Symposium on Bioorganic Chemistry. The program, which included twenty-five plenary lectures plus poster presentations, covered broad areas: I. Mimics of biological systems. II. The chemistry of natural products of biological importance, and III. The chemistry of biological macromolecules. Once it was decided to continue IUPAC symposia under this title, it was not our intent to wait eight years until we scheduled the second in the series, but circumstances beyond our control dictated this delay. In the interim, other international symposia, several under IUPAC sponsorship, including those on The Chemistry of Natural Products, have included some of the material of bioorganic chemistry.

Other conferences have also included such material: for example, the irregularly scheduled International Roundtables on Nucleosides, Nucleotides, and their Biological Applications and the FECHEM Conferences on Heterocycles in Bioorganic Chemistry - Hungary, 1984 and Belgium, 1986. In the United Stated there has been a biennial series of symposia on "Chemistry as a Life Science," cosponsored by Rutgers University, the New Jersey Section of the American Chemical Society, and several pharmaceutical companies, and a 1991 Robert A. Welch Foundation Conference on Chemical Research, "Chemistry at the Frontiers of Medicine." The second Gordon Research Conference on Bioorganic Chemistry is being held this month in New Hampshire.

The organizers of this Second International Symposium on Bioorganic Chemistry have arranged the program in a very imaginative and effective manner. The announced general areas to be covered include the following:

1. Enzyme Functions and Modelings

2. Biological and Artificial Receptors

3. Biosynthetic Pathways and Biologically Active Molecules

4. Organic Chemistry Aspects of Genetic Engineering

5. Biopolymers and Artificial Supramolecular Assemblies

6. Simulation of Biochemical Processes. 
As you will see in your scientific programs, there are three plenary lectures at the beginning of the week to stimulate us and to exemplify the breadth and scope of the research to be described, and there are two exciting plenary lectures on the final afternoon to ensure that we remain in residence throughout the four days:

\author{
Abzymes and Enzymes \\ S. J. Benkovic, The Pennsylvania State University, \\ University Park \\ Rational Design of Sequence-Specific DNA Ligands for Artificial \\ Control of Gene Expression \\ C. Hélène, Muséum National d'Histoire Nature111e, France \\ Enzymes of B-Lactam Biosynthesis \\ J. E. Baldwin, University of Oxford, U.K. \\ Molecular Recognition by Artificial Receptors \\ D. N. Reinhoudt, University of Twente, The Netherlands \\ Bioorganic Studies of Receptors with Philanthotoxine Analogs \\ K. Nakanishi, Columbia University, New York.
}

On Tuesday, Wednesday, and Thursday mornings there are parallel sessions of invited lectures. The remaining time is devoted to oral presentations and poster sessions. Among many topics we shall learn about are the following:

Enzyme-coenzyme interaction and aclivation.

Catalysis with adsorbed enzymes and enzymatic conversions in organic solvents.

Enantioselective and diastereoselective enzyme reactions, along with enzyme-induced racemizations.

Requirements for million-fold rate acceleration in enzyme catalysis.

Site-specific incorporation of nonnatural amino acids into proteins and the consequences thereof on binding and activity.

Catalytic antibodies.

$\alpha$-Helix bundle polypeptides.

DNA triple helices.

Chemical and photochemical cleavage of DNA.

Mimics of biological electron-transfer systems.

Neurotoxins and neuronal receptors.

From all this, we can conclude that the enterprise of bioorganic chemisty is progressing at an accelerated pace and in ever broadening scope. We offer our sincere thanks to Professors Yukito Murakami and Makoto Takagi for treating us to such a lively and stimulating program.

\title{
REFEREHCES
}

1. B. Witkop Science 162, 318-326 (1968).

2. See: B. Zerner Bioorg. Chem. 20, 269-284 (1992).

3. N. J. Leonard Biopolymers 24, 9-28 (1985). 